for searching and reviewing the evidence on CAM in cancer.

doi: $10.1016 /$ j.ctim.2004.07.018

\section{The drivers for complementary therapy use in cancer}

Jessica Corner*, Jackie Harewood, Sian MaslinProthero, George Lewith, Jane Maher, Teresa Young, Clare Gwilliam

School of Nursing, University of Southampton, Midwifery, Nightingale Building, University Road, Highfield, Southampton S017 1BJ, UK

*Corresponding author.

Studies of complementary and alternative therapy (CAM) use among patients with cancer suggest that these are used by a substantial proportion of patients, although the figure varies considerably across studies. We do know that CAM use is more common among people who are younger, female, or of higher socio-economic status and education background. Users may be more likely to be anxious or depressed, have a high belief in the efficacy of CAM and a corresponding dissatisfaction with conventional medicine. CAM is also known to be used as an active method of coping with conventional cancer treatment. However, studies examining the use of CAM among people with cancer have limitations. Studies have relied heavily on survey methods which, although useful, tell us little about the complex issues surrounding CAM use. Distinct motivations have been identified such as to treat the disease or prevent cancer recurring, or as an adjunct to conventional cancer treatment to maximise quality of life or to assist in returning to normal life after treatment.

We are undertaking a study of the use of CAM among people undergoing cancer treatment funded by the NHS R\&D Programme. We are in the process surveying a sample of 300 patients from twocancer centres, stratified to represent the UK incidence of cancer. We are also interviewing, using qualitative biographic narrative methods, a sub-sample of 40 CAM users and 10 nonusers. The study is ongoing with 184 questionnaires completed to date. Early findings from the survey suggest that there are three types of CAM 'user'. Firstly, individuals who used CAM prior to their cancer diagnosis and continue to use CAM afterwards. Secondly, individuals who did not use CAM prior to their diagnosis but have used it since, some of these individuals have used CAM because it has been offered as part of their treatment and support. Thirdly, people who used CAM prior to their diagnosis but have stopped since because of concerns over the safety or appropriateness of combining CAM with conventional cancer treatment.

It is possible to identify a number of drivers for CAM use in cancer, both those that act to promote CAM use, but also those that prevent or inhibit CAM use. These include personal motivation, accessibility and availability of CAM, financial resources, the desire to actively manage one's illness, availability of evidence about the value and safety of CAM as an adjunct to cancer treatment, and a lack of informed guidance for patients.

doi: 10.1016/j.ctim.2004.07.019

Research complementary and alternative therapy-an oncologist's view

\section{E.J. Maher \\ Macmillan Cancer Relief, 89 Albert Embankment, London SE1 7UQ, UK}

As an oncologist, I became involved in complementary and alternative treatments (CAMs) because my patients were using them. ${ }^{1}$ For this reason our support care in the Lynda Jackson Macmillan Centre at Mount Vernon Cancer Centre included CAMs in the services offered. Between 1993 and 1998, we established a service model housed in a new building, including supportive listening, high quality information, "touch, talk and time" therapies and psychological support from psychologists and psychiatrists. We were not alone, as most cancer centres in the UK now employ at least one CAM therapist. A key aspect of the success of LJMC was its position as part of a large cancer centre serving 5000 new patients with access to 25 oncologists in 15 district general hospital with all their networks and nearly 50 clinical scientists. This allowed exchange of learning between orthodox and complementary practitioners and service developers and researchers. Therapies were introduced step-by-step focussing on developing a shared language, understanding the therapy from a range of perspectives and testing the use of outcome measure and study designs which produced data meaningful to all stakeholders.

During this work, we recognised the importance of collaboration with academic centres with a wide range of different expertise, of using the tools of education and information as well as research to change practice but also producing peer reviewed publications. We recognised the need to work to- 